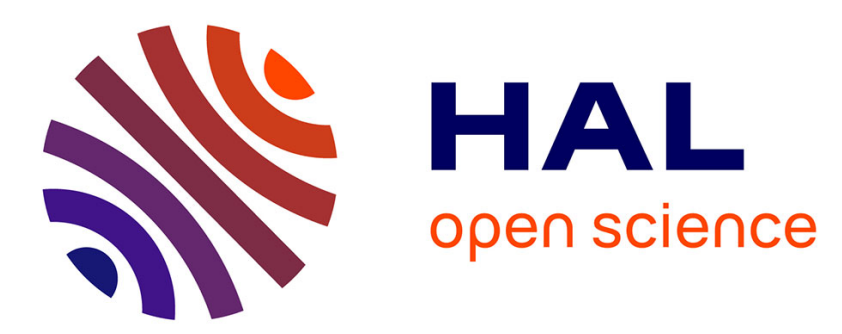

\title{
L'intimidation. Retour sur la campagne de presse qui a mené à l'enterrement des rapports
}

\author{
Fabrice Dhume
}

\section{To cite this version:}

Fabrice Dhume. L'intimidation. Retour sur la campagne de presse qui a mené à l'enterrement des rapports. Migrations Société, 2014, Intégration : une "refondation" enlisée, 26 (155), p.131-152. halshs01421731

\section{HAL Id: halshs-01421731 \\ https://shs.hal.science/halshs-01421731}

Submitted on 22 Dec 2016

HAL is a multi-disciplinary open access archive for the deposit and dissemination of scientific research documents, whether they are published or not. The documents may come from teaching and research institutions in France or abroad, or from public or private research centers.
L'archive ouverte pluridisciplinaire HAL, est destinée au dépôt et à la diffusion de documents scientifiques de niveau recherche, publiés ou non, émanant des établissements d'enseignement et de recherche français ou étrangers, des laboratoires publics ou privés. 


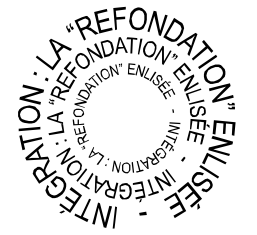

\section{L'INTIMIDATION : RETOUR SUR LA CAMPAGNE DE PRESSE QUI A MENÉ À L'ENTERREMENT DES RAPPORTS}

Fabrice DHUME *

La polémique qui a suivi la réception des rapports sur la II refondation de la politique d'intégration $1{ }^{1}$, à la fin 2013 , a abouti au retrait gouvernemental global et immédiat de l'initiative politique et a conforté la nature de question "sensible" du thème. Les rapports ont été présentés comme une "l menace pour les valeurs et les intérêts de la société ${ }^{2}{ }^{2}$, dans une stratégie spécifique de dramatisation et de disqualification, soit une démonstration de force visant à exclure du champ du dicible et du représentable certaines questions, positions ou propositions.

Par-delà, et à travers une polémique opportune pour certains groupes (échéances électorales, stratégie économique d'une presse en perte de vitesse...), on peut supposer que cette intimidation visait à maintenir le "consensus indicible $\|^{3}$ que recouvre le thème de l'intégration en France. Celui-ci repose sur une combinaison idéologique

* Sociologue, chercheur à l'Institut social et coopératif de recherche appliquée (ISCRA), Montpellier.

1. TUOT, Thierry, La grande Nation : pour une société inclusive. Rapport au Premier ministre sur la refondation des politiques d'intégration, Paris : Cabinet du Premier ministre, $1^{\text {er }}$ février 2013 , 100 p., http://www.ladocumentationfrancaise.fr/rapports-publics/134000099 ; DHUME, Fabrice ; HAMDANI, Khalid, Vers une politique française de l'égalité. Rapport du groupe de travail "Mobilités sociales" dans le cadre de la "Refondation de la politique d'intégration", novembre 2013, 93 p., http://www.ladocumentationfrancaise.fr/var/storage/rapports-publics/134000758/0000.pdf ; LAMARRE, Chantal ; MAFFESSOLI, Murielle, Refondation de la politique d'intégration. Groupe de travail "Connaissance - reconnaissance", rapport remis en novembre 2013, 62 p., http://www.ladocumentationfrancaise.fr/var/storage/rapports-publics/134000756/0000.pdf ; MADELIN, Bénédicte ; GENTIAL, Dominique ; GOYAUX, Nathalie ; BOUILLE, Ambrosini, Refonder la politique d'intégration : rapport du groupe Protection sociale remis le 29 octobre 2013, 42 p., http://www.ladocumentationfrancaise.fr/var/storage/rapports-publics/134000759/0000.pdf ; KHIROUNI, Chaynesse; TALLAND, Chantal, L'habitat, facteur d'intégration, rapport remis en octobre 2013, 32 p., http://www.ladocumentationfrancaise.fr/var/storage/rapports-publics/ 134000757/0000.pdf ; BOUBEKER, Ahmed ; NOËL, Olivier, Faire société commune dans une société diverse, rapport remis le 5 novembre 2013, $47 \mathrm{p}$.

2. COHEN, Stanley, Folk devils and moral panics, London : Mac Gibbon and Kee Publishers, 1972, $224 \mathrm{p}$.

3. WEIL, Patrick, La France et ses étrangers : l'aventure d'une politique de l'immigration, 19381991, Paris : Éd. Calmann-Lévy, 1991, 403 p. 
répondant notamment au succès de la rhétorique d'extrême droite ${ }^{4}$ : la construction d'une population ("immigrés", "musulmans"...) en tant que "problème", au regard d'une définition de la situation sous l'angle de "la nation en danger". Comment cet épisode polémique s'est-il construit? Quels en ont été les ressorts pratiques et stratégiques? Et que nous dit-il finalement de l'état du consensus "républicain" sur l'intégration"?

\section{Une analyse des discours médiatiques}

Les discours médiatiques qui ont donné corps à cette polémique ont usé de quelques grands procédés et ressorts de dramatisation et de disqualification. Le type de média influant sur la forme des discours, nous distinguerons a priori les médias d'information et les médias d'opinion (tribunes, blogs, etc.). L'intérêt de mobiliser les deux est de prendre la mesure de la continuité argumentaire, par-delà ou à travers une radicalisation éventuelle des formes d'un média à l'autre. Relevons une bizarrerie : les journalistes mêmes qui disent des rapports qu'ils "prônent la désintégration de la nation $~ "^{5}$ concluent en appelant à un "débat" sur la question". "Le débat sur l'intégration est décisif, il n'aura donc pas lieu ", ose le magazine Causeur en titrant "Français contre Français $1{ }^{7}$. On pourrait se demander : pourquoi débattre si la situation est si grave qu'on le dit? L'appel au "l débat "I semble relever de la contrainte dite "démocratique", alors que l'enjeu des discours est, à l'inverse, de faire taire certaines voix.

L'analyse présentée par la suite porte sur les discours médiatiques commentant directement les cinq rapports concernés. Le corpus comprend 215 textes publiés entre le 9 décembre 2013 (avant le départ de la polémique) et le 18 février 2014 (après la publication de la Feuille de route du gouvernement ${ }^{8}$ ). Bien que non exhaustif, ce

4. Cf. BONNAFOUS, Simone, L'immigration prise aux mots: les immigrés dans la presse au tournant des années 80, Paris : Éd. Kimé, 1991, 301 p. Cela permet en partie de comprendre pourquoi il apparaît à certains observateurs de la polémique que « sous couvert de consensus républicain, réputé apolitique ", la politique du gouvernement " de gauche " est indexée aux logiques « de droite». Voir FASSIN, Éric, "Intégration : un consensus de droite", blogs.mediapart.fr/ blog/eric-fassin, 19 décembre 2013.

5. RIOUFOL, Ivan, "Intégration : l'État perméable à l'islam politique", blog.lefigaro.fr/rioufol/, 16 décembre 2013.

6. Ivan Rioufol, sur France Ô, le 25 janvier 2014.

7. LÉVY, Élisabeth, "Français contre Français. Le débat sur l'intégration est décisif, il n'aura donc pas lieu”, http://www.causeur.fr, 6 janvier 2014.

8. PREMIER MINISTRE, Politique d'égalité républicaine et d'intégration : feuille de route du gouvernement, Paris, 11 février 2014, 21 p., http://www.cofrimi.com/images/PDF/feuille_route_ politique_degalite_republicaine_dintegratio-1.pdf 
corpus s'en approche à partir d'une recherche par mots-clés ${ }^{9}$ répétée et enrichie par les textes mis en liens par les rédactions. II exclut les émissions télévisées et radiodiffusées, mais inclut les écrits des sites d'information des chaînes (tfl f.fr, rtl.fr, bfmtv.com, arte.tv, etc.).

Pour l'analyse, ce corpus est scindé en deux ensembles : 100 articles d'information, tirés de sites d'information, de quotidiens nationaux ou régionaux, d'hebdomadaires ou de revues français, qui, tous titres confondus, constituent le cœur de l'analyse ; 115 textes tirés des médias d'opinion (tribunes, chroniques, blogs ou communiqués de presse) la complètent. Nous recourons à une approche mi-thématique, michronologique : pour les principaux ressorts de disqualification identifiés, nous partirons de la présentation du Figaro, qui a démarré la campagne de presse, avant de voir le traitement dans les textes d'information, puis dans ceux d'opinion. Nous terminerons en proposant une hypothèse d'interprétation de la forme de cette polémique.

Nous ne chercherons pas ici à juger de la légitimité ou de l'opportunité des critiques qui ont été adressées aux rapports, d'autant que, sauf rares exceptions, ce n'est pas de leurs limites objectives qu'il a été "discuté", et ce n'est pas non plus des textes en eux-mêmes. On travaillera surtout sur la face formelle de la polémique pour dégager les enjeux policiers sous-jacents à cette stratégie de disqualification. À ce sujet, nous suivrons Jacques Rancière lorsqu'il distingue au sein du politique la politique, qui II consiste dans le jeu des pratiques guidées par la présupposition de l'égalité de n'importe qui avec n'importe qui et par le souci de la vérifier 11 , et la police, qui désigne les logiques et activités de maintien d'un ordre donné, qui exclut toujours certaines voix ${ }^{10}$.

\section{Le déchaînement}

L'affaire est entendue : le point de départ de la médiatisation est la série d'articles publiés par Le Figaro du 12 décembre 2013, signés par Stéphane Kovacs ${ }^{11}$. Jusque-là, la publication discrète des rapports des groupes de travail sur le site du Premier ministre était restée confidentielle. Quelques réseaux spécialisés — notamment dans le domaine scolaire - en avaient répercuté l'information ou publié des extraits. Michèle Tribalat en avait fait une critique dès le 9 décembre

9. "Refondation + intégration" ; "rapport + intégration" ; "rapport + nom des auteurs".

10. RANCIÈRE, Jacques, Aux bords du politique, Paris : Éd. La Fabrique, 1998, 189 p. (voir p. 112).

11. KOVACS, Stéphane, "Intégration : un rapport choc”, www.lefigaro.fr,12 décembre 2013. 
$2013^{12}$, inaugurant plusieurs thèmes de la polémique et en constituant une base remarquée ${ }^{13}$. Mais le dossier du Figaro efface cette lente diffusion au profit d'un "coup" médiatique reposant sur une belle manipulation journalistique. Le premier procédé est l'unification de l'objet et sa construction comme menace. Dès la une, les cinq rapports sont nommés comme un tout (in)cohérent : "Un rapport " ${ }^{14}$. Cela aura pour effet durable d'effacer des divergences importantes entre textes ${ }^{15}$, d'évacuer la cohérence interne à chacun et d'imputer à l'objet un "foisonnement " qui devient lui-même un thème de disqualification : les médias stigmatiseront un "rapport fourre-tout " ${ }^{16}$, un " bric-àbrac de propositions $\|^{17}$, des "propositions disparates dont le seul point commun est de favoriser le communautarisme $\|^{18}$ ou une " bouillie en cinq parties $11^{19}$. Pour cela, la journaliste Stéphane Kovacs a pioché des formules dans trois des cinq rapports ${ }^{20}$ : les extraits, lapidaires, sont décontextualisés; le sens de la formule est privilégié, transformant de longues analyses en une poignée de slogans : " $\grave{A}$ coup de "vivre ensemble égalitaire", de "changement de paradigme", de "production de possibles", tout est mis en place pour "faire de l'en-commun" $11^{21}$.

12. TRIBALAT, Michèle, "Intégration : les 5 rapports qui poussent la France sur la voie du multiculturalisme choisi sans le dire trop haut", www.atlantico.fr, 9 décembre 2013.

13. CRAPEZ, Marc, "Les dessous du rapport sur l'intégration", www.contrepoints.org, 16 décembre 2013.

14. Ibidem.

15. Élise Vincent et Stéphanie Le Bars, journalistes du Monde, découvriront rétrospectivement la principale ligne de clivage des rapports, entre un discours intégrationniste et ne tenant pas compte de la couleur de la peau, un discours antidiscriminatoire et, enfin, des positions plus multiculturalistes. Voir VINCENT, Élise ; LE BARS, Stéphanie, "La polémique autour de l'intégration révèle l'embarras de l'exécutif sur le sujet", www.lemonde.fr, 14 décembre 2013.

16. GUIROUS, Lydia, "Laïcité inclusive : la France des Lumières brûle", www.huffingtonpost.fr, 13 décembre 2013.

17. PROUTEAU, Thomas, "Le rapport sur I'Intégration, une parano et un énorme gâchis", www.slate.fr, 19 décembre 2013.

18. BOURGUEREAU, Jean-Marcel, "Intégration, le rapport qui divise", www.larepubliquedespyrenees.fr, 14 décembre 2013.

19. CONSIGNY, Charles, "Consigny : ce que dit vraiment le rapport sur l'intégration", www.lepoint.fr, 16 décembre 2013.

20. Soit ceux des groupes "Connaissance-reconnaissance", "Faire société pour une égalité réelle", et "Mobilités sociales". Sur l'ensemble du corpus, le rapport du groupe de travail "Habitat" n'est cité que dans $2 \%$ des articles d'information. Dans sa critique inaugurale, Michèle Tribalat justifie ainsi ce silence : "Signalons, pour ne plus en reparler, le rapport sur l'habitat rendu illisible par le langage, la syntaxe et l'orthographe". La disqualification par la forme scolaire a ici un effet rédhibitoire : même pas la peine d'en discuter. Car l'enjeu est ailleurs. Voir TRIBALAT, Michèle, "Intégration : les 5 rapports qui poussent la France sur la voie du multiculturalisme choisi sans le dire trop haut", www.atlantico.fr, 9 décembre 2013.

21. KOVACS, Stéphane, "Assumer la dimension 'arabe-orientale' de la France", www.lefigaro.fr, 12 décembre 2013. 
Enfin, l'accent est mis sur les préconisations - alors que plusieurs des rapports insistent sur l'analyse du problème - elles aussi traitées sous la forme d'un pot-pourri tiré de deux-trois rapports et décontextualisées pour susciter un sentiment d'incongruité, "idéalistes ", " provocatrices " ou " iconoclastes ", concluront les autres médias. Le titre impute d'entrée de jeu une brutalité et une radicalité : " Un rapport choc I", "I sommés "d"opérer un déplacement [...]", les experts font carrément demi-tour $11^{22}$.

De ces procédés rhétoriques - usuels dans la mise en scène journalistique - découle une longue série de brouillages et de confusions ${ }^{23}$ qui servira la stratégie polémique en plaçant d'emblée hors débat toute nuance, et finalement la discussion même des rapports. Le choix devient binaire : pour ou contre... mais la présentation ne laisse guère le choix.

Par la suite, la principale sinon l'unique référence des médias ${ }^{24}$ est le discours du Figaro, répercuté par les agences de presse et jusque dans l'article de Wikipédia récemment consacré aux "I Politiques d'intégration en France $11^{25}$. La référence à ce journal va de pair avec la validation et le renforcement de l'incrimination d'un objet-problème, qui se confond vite dans un thème-problème (l'"intégration") identifié à la conflictualité. Le rapport de Thierry Tuot remis en février $2013^{26}$,

22. KOVACS, Stéphane, "Intégration : un rapport choc", www.lefigaro.fr, 12 décembre 2013.

23. Par exemple, le thème "du" rapport unique conduit des journalistes à croire qu'« à la baguette de ce texte, on retrouve Thierry Tuot " (JUHEL, Chloé, "L'intégration, nouvelle épine dans le pied de Hollande", www.lecourrierdelatlas.com, 16 décembre 2013), idée répercutée dans divers médias d'opinion (PARTI DE LA BANLIEUE, "Communiqué de presse : suite au rapport sur la refondation des politiques d'intégration", lepartidelabanlieue.fr, 14 décembre 2013; BOCHEL GUÉGAN, Dom, "Rapport sur l'intégration : un écran de fumée digne du débat sur l'identité nationale", leplus.nouvelsobs.com/contribution, 14 décembre 2013 ; LELONG, Guillaume, "L'inexcusable Rapport Intégration", guillaumelelong.fr, 15 décembre 2013), ou à confondre avec le rapport Blanchard sorti en octobre 2013 (BLANCHARD, Pascal, Histoires, patrimoine et mémoires dans les territoires de la politique de la ville : rapport au ministre délégué à la Ville, François Lamy, octobre 2013, 65 p., http://www.ville.gouv.fr/IMG/pdf/rapport_- histoires patrimoine_et_memoires_dans_les_territoires_de_la_politique_de_la_ville.pdf). Voir LËMONONIER, Marie; MĀRTIN, Julien, "Intégration : comment Ayrault s'est pris les pieds dans le voile", tempsreel.nouvelobs.com, 22 décembre 2013.

24. À l'exception notable de L'Express qui rend compte du rapport Faire société commune dans une société diverse (MISSIR, Marie-Caroline, "Rapport sur l'intégration : vers une laïcité de 'compromis'”, www.lexpress.fr, 13 décembre 2013), puis quelques jours plus tard de Mediapart, qui traite du rapport Vers une politique française de l'égalité (DELAPORTE, Lucie, "'Intégration' : ce rapport déjà enterré et que personne n'a lu", www.mediapart.fr, 18 décembre 2013).

25. https://fr.wikipedia.org/wiki/Politiques_d\%27int\%C3\%A9gration_en_France, consulté le 20 juin 2014.

26. TUOT, Thierry, La grande Nation : pour une société inclusive. Rapport au Premier ministre sur la refondation des politiques d'intégration, Paris : Cabinet du Premier ministre, $1^{\text {er }}$ février 2013 , 100 p., http://www.ladocumentationfrancaise.fr/ var/storage/rapports-publics/134000099/0000.pdf 
déjà, avait été qualifié de $\|$ rapport au bazooka $\|^{27}$ ou $\|$ au vitriol $\|^{28}$, témoignant d'une logique de guerre ou d'un fantasme de défiguration, par lesquels le thème est abordé : "I La question de l'intégration " est assimilée à " une bombe " potentielle ${ }^{29}$, "l de la nitroglycérine $\|^{30}$, " ultrasensible $\|^{31}$. Le Figaro aurait tout au plus $\|$ mis le feu aux poudres $\|^{32}$.

L'"intégration" s'impose comme une "question sensible", justifiant d'y toucher le moins possible et donc de maintenir un statu quo présenté comme un consensus. À de rares exceptions - comme RFI, qui met " en cause le quotidien Le Figaro " ${ }^{33}$ — les médias imputent aux rapports la charge polémique, et donc la responsabilité de la rupture du consensus. Le Journal du Dimanche titre "I 276 pages, 276 polémiques ${ }^{34}$, tandis que le titre du Figaro devient littéralement le nom de l'objet : " Le rapport-choc $\|^{35}$, "rapport polémique $\|^{36}$, "rapport

27. FOUTEAU, Carine, "Le rapport qui dézingue la politique d'intégration", www.mediapart.fr, 8 février 2013.

28. "Vives réactions après l'exhumation d'un rapport sur l'intégration”, www.liberation.fr, 13 décembre 2013.

29. "Le rapport sur l'intégration commandé par Matignon fait polémique", www.francetvinfo.fr 13 décembre 2013.

30. AMAR, Cécile, "Le rapport qui embarrasse l'exécutif", www.lejdd.fr, 15 décembre 2013 ; REVAULT d'ALLONNES, David, "Le faux pas de M. Ayrault sur l'intégration aiguise les appétits de ses rivaux", www.lemonde.fr, 16 décembre 2013 ; VENTURA, Alba, "Rapport sur l'intégration : Ayrault s'est tiré une balle dans le pied", www.rtl.fr, 16 décembre 2013.

31. VERNET, Henri, "Intégration: $67 \%$ des Français opposés à la discrimination positive", leparisien.fr, 7 février 2014 ; LOZÈS, Patrick, "Flou sur le voile à l'école : le gouvernement en apprenti-sorcier", www.huffingtonpost.fr, 16 décembre 2013.

32. "Un rapport sur l'intégration enflamme les échanges entre gouvernement et UMP", leparisien.fr, 13 décembre 2013 ; “Intégration : Copé fait monter la polémique, Ayrault réplique, Le Pen en embuscade", www.lexpress.fr, 13 décembre 2013 ; HOFFNER, Anne-Bénédicte, "Cinq rapports préconisant de 'repenser l'intégration' suscitent un tollé", www.la-croix.com, 13 décembre 2013; HAMCHICHE, Imene, "Intégration : le rapport qui divise", movilibercampus.fr, 14 décembre 2013 ; "Le rapport sur l'intégration a fâché François Hollande", www.lanouvellerepublique.fr, 15 décembre 2013 ; "Rapport sur l'intégration : Ayrault réfute tout couac", tempsreel.nouvelobs.com, 16 décembre 2013 ; JUHEL, Chloé, "L'intégration, nouvelle épine dans le pied de Hollande", www.lecourrierdelatlas.com, 16 décembre 2013 ; JUHEL, Chloé, "Le gouvernement tourne le dos à l'intégration”, www.lecourrierdelatlas.com, 23 décembre 2013.

33. "Rapport sur l'intégration: I'UMP dénonce un risque de communautarisme", www.rfi.fr, 13 décembre 2013.

34. FLEURY, Adeline ; QUENET, Marie, "Rapport sur l'intégration : 276 pages, 276 polémiques", www.lejdd.fr, 14 décembre 2013.

35. "Intégration : les propositions du rapport-choc", www.lepoint.fr, 13 décembre 2013 ; DHOLLANDEMONNIER, Victor, "Intégration : ce rapport choc remis à Ayrault", www.europe1.fr, 13 décembre 2013 ; "Un rapport choc préconise d'autoriser le voile à l'école", journaldumusulman.fr, 13 décembre 2013 ; LANGLET, Julien, "Le rapport-choc qui veut refonder l'intégration en France", www.franceinfo.fr, 13 décembre 2013; "Le rapport choc sur l'intégration”, www.arte.tv, 14 décembre 2013.

36. "Un rapport polémique sur l'intégration oblige François Hollande à intervenir", www.franceculture.fr, 13 décembre 2013 ; "Intégration : ce que préconise le rapport polémique remis à Ayrault", Ici.tf1.fr, 14 décembre 2013 ; HAMCHICHE, Imene, "Intégration: le rapport qui divise", movil.ibercampus.fr, 14 décembre 2013 ; REVAULT d'ALLONNES, David, "Le faux pas de M. Ayrault sur l'intégration aiguise les appétits de ses rivaux", www.lemonde.fr, 16 décembre 2013. 


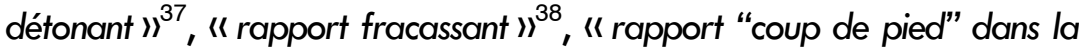
fourmilière de l'intégration $\|^{39}$, " rapport litigieux $\|^{40}$, " le rapport qui

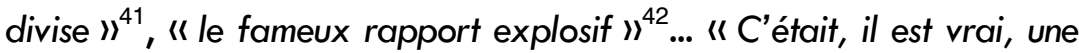
bombe [...] cet étrange rapport $"$, conclura L'Opinion ${ }^{43}$.

Dans les textes d'opinion, cette dramaturgie de l'objet-menace est accentuée par des représentants politiques en concurrence dans la prétention à incarner "la République ". À l'"l alerte aux pyromanes" face à " une arme de destruction massive destinée à pulvériser notre modèle politique social et culturel " lancée par le journaliste Pierre Beylau ${ }^{44}$ répond " l'alarme " de politiciens de droite qui se disputent la place de pompier-en-chef : François Fillon " alerte $\|^{45}$, le président du Parti chrétien-démocrate, Jean-Frédéric Poisson, "tire le signal d'alarme $1{ }^{46}$, etc.

"I La France est en train de bruler ces [sic] papiers d'identité et si cela continue, elle se retrouvera comme un clandestin dans le concert des nations 11, affirme Lydia Guirous, de l'Union des démocrates et indépendants (UDI) ${ }^{47}$. Les blogs suivent, chacun croyant analyser en la matière "I soit une provocation sociétale, soit un rapport explosif qui sème la division et la zizanie $1{ }^{48}$. L'extrême droite ne dit pas autre

37. T.d.L., "Intégration : ce que contient le rapport détonant remis à Matignon", leparisien.fr, 13 décembre 2013 ; LEMONNIER, Marie ; MARTIN, Julien, "Intégration : comment Ayrault s'est pris les pieds dans le voile", tempsreel.nouvelobs.com, 22 décembre 2013.

38. "Un rapport fracassant sur l'intégration préconise l'autorisation du voile à l'école", oumma.com, 13 décembre 2013.

39. JUHEL, Chloé, "Un rapport 'coup de pied' dans la fourmilière de l'intégration”, www.lecourrierdelatlas.com, 13 décembre 2013

40. "Rapport sur l'intégration: Ayrault réfute tout couac", tempsreel.nouvelobs.com, 16 décembre 2013 ; "Rapport sur l'intégration: Matignon encore la cible de critiques", www.leparisien.fr, 16 décembre 2013.

41. FLEURY, Adeline ; QUENET, Marie, "Rapport sur l'intégration : 276 pages, 276 polémiques", www.lejdd.fr, 14 décembre 2013.

42. L. B., "Rapport sur l'intégration: Hollande ignorait sa mise en ligne", www.bfmtv.com, 14 décembre 2013 ; L. B., "Désir : l'interdiction du voile à l'école pas remise en cause", www.bfmtv.com, 15 décembre 2013; ROVAN, Anne, "Rapport sur l'intégration: Royal tacle la 'méthode' Matignon", www.lefigaro.fr, 16 décembre 2013.

43. MONTVALLON, Dominique de; HOUCHARD, Béatrice, "L'incroyable histoire du vrai-faux rapport sur l'intégration", www.lopinion.fr, 15 décembre 2013.

44. BEYLAU, Pierre, "Intégration : alerte aux pyromanes", www.lepoint.fr, 13 décembre 2013.

45. FILLON, François, "Intégration ou désintégration ?”, blog-fillon.com, 13 décembre 2013.

46. POISSON, Jean-Frédéric, "Rapport sur l'intégration : des propositions terrifiantes", lepcd.fr, 13 décembre 2013.

47. GUIROUS, Lydia, "Laïcité inclusive: la France des Lumières brûle", www.huffingtonpost.fr, 13 décembre 2013.

48. "Rapport intégration-Immigration... Mais qu'ils consultent donc le Peuple souverain, Bon Dieu !", switchie5.wordpress.com, 13 décembre 2013. 
chose : "I Les conclusions des groupes de travail [...] apparaissent comme une très grave provocation. Leur mise en œuvre signifierait l'abandon définitif du modèle républicain, la mise en place d'une société ultracommunautarisée et divisée. Elle constituerait une véritable déclaration de guerre aux Français qui eux réclament l'arrêt de la politique d'immigration massive et la réaffirmation de nos lois républicaines et de nos valeurs $11^{49}$. De ce fait, il y a bien continuité et unité d'approche entre les discours de la majeure partie des médias d'information et ceux des politiciens de la droite, puis des blogs (sans parler des réactions de lecteurs qui se mettent au diapason). Tous se retrouvent dans la mise en passion (scandale) et le traitement à charge (menace) des rapports. $\mathrm{Si}$ les médias d'information dénoncent la rupture du consensus, les médias d'opinion les chargent de l'effet passionnel, et tous ou presque veulent y voir l'expression d'une " idéologie ॥.

À l'opposé, l'"intégration républicaine" est présentée comme le modèle de raison, celui d'une société pacifiée et pacificatrice. L'argument de la laïcité - " valeur si typiquement française $\|^{50}$, écrit le Journal du Dimanche - est mobilisé de façon métaphorique: l'idée de "neutralité" sert d'équivalent à celle de paix sociale, opposée comme principe aux " fauteurs de trouble "). L'exemple type est la loi du 15 mars 2004 sur le voile, qui, lit-on, II s'est imposée comme un texte plutôt consensuel, à droite comme à gauche $\|^{51}$, " pacifie justement les écoles et fait qu'un enfant est considéré comme un élève, pas comme un religieux $~^{52}$, " qui fut si difficile à mettre en œuvre et qui semble aujourd'hui avoir laissé les esprits pacifiés $1^{53}$. Et les politiques de prendre le relais, comme Ségolène Royal "d'insister : il ne faut "absolument pas" reprendre le débat sur le foulard islamique à l'école qui "a déjà eu lieu, a été bien tranché", "est efficace et a été compris par l'ensemble des communautés" $1{ }^{54}$.

49. LE PEN, Marine, "Communiqué de presse de Marine Le Pen, Présidente du Front National", www.frontnational.com, 13 décembre 2013.

50. AMAR, Cécile, "Le rapport qui embarrasse l'exécutif", www.lejdd.fr, 15 décembre 2013.

51. LE BARS, Stéphanie, "Revenir sur le voile à l'école : une proposition iconoclaste et inutile?", www.lemonde.fr, 13 décembre 2013.

52. PHAM-LÊ, Jérémie, "Intégration : 'Ce rapport part du principe que la France est coupable et raciste'”, www.lexpress.fr, 13 décembre 2013.

53. SINCLAIR, Anne, "Le rapport sur l'intégration : un beau ratage", huffingtonpost.fr, 16 décembre 2013.

54. "Rapport sur l'intégration : Ayrault réfute tout couac", tempsreel.nouvelobs.com, 16 décembre 2013. 
Ce grand consensus est en même temps démenti par les débats entre lecteurs... mais l'important n'est pas là, car l'imputation aux rapports de la polémique permet de marginaliser les voix discordantes, et donc le débat lui-même. Comme le note SaphirNews, II la polémique ne donne pas lieu à un débat de fond car à gauche comme à droite, on s'accorde tous pour dire que le voile n'a pas sa place à l'école $1{ }^{55}$. Ainsi Stéphanie Le Bars, du Monde, justifie-t-elle de révoquer la question du voile : "Cette proposition est d'autant plus iconoclaste, pour ne pas dire inutile, qu'elle ne correspond guère à une quelconque demande sociale ou politique [...]. Aujourd'hui, seuls les milieux militants du Comité 15 mars et Libertés, proches de l'Union des organisations islamiques de France (UOIF) ou du Collectif contre l'islamophobie en France (CCIF), dénoncent toujours une loi discriminatoire $11^{56}$. Le consensus c'est la Raison; et la polémique serait le jeu des "islamistes", dont la voix n'est que bruit ("militant"). Et les lecteurs de commenter ce bien-entendu : " Demander un rapport à ce genre de personne [un "islamiste"] dans le cadre de notre République encore un peu éclairée [...] c'est effectivement totalement irresponsable $11^{57}$.

\section{La symbolique de l'affrontement}

D'emblée, Le Figaro dessine un affrontement et distribue les rôles. La narration du journal entremêle une factualité (on cite les noms des groupes, on donne des dates, on met des guillemets, etc.) et un registre sémantique de l'agressivité et de l'imposition ("sommés ", "l exhorte ", " stigmatise ", "l assènent ", " haro sur "), soutenu par des points d'exclamation. La posture morale de la journaliste est ainsi projetée sur l'objet : " Autorisation du voile à l'école, création d'un "délit de harcèlement racial" et d'une "Cour des comptes de l'égalité", ces propositions détonantes vont-elles être reprises par le gouvernement? Le Premier ministre, en tout cas, "salue la grande qualité" de ces travaux $1{ }^{58}$.

La conclusion sous forme de mise en tension résonne comme une mise au défi du gouvernement et comme un appel à l'opposition. Elle prépare le terrain à l'affrontement politicien en même temps qu'à

\footnotetext{
55. "Esther Benbassa: le rapport sur l'intégration 'sacrifié sur l'autel de l'islamophobie'”, SaphirNews.com, 16 décembre 2013.

56. LE BARS, Stéphanie, "Revenir sur le voile à l'école : une proposition iconoclaste et inutile ?", www.lemonde.fr, 13 décembre 2013. Le gras est de notre fait.

57. Ibidem.

58. KOVACS, Stéphane, "Intégration : un rapport choc", www.lefigaro.fr, 12 décembre 2013.
} 
une rhétorique funèbre sur l'"enterrement" des rapports que la journaliste soldera deux mois plus tard : "Voilà un rapport qui a fait pschitt ! $11^{59}$. Tandis que les rapports sont minorisés, le journal majore son propre rôle : à l'instar d'un "scoop", la journaliste parle de rapports qui " viennent d'être publiés $\|^{60}$, alors que l'article paraît presque un mois après leur mise en ligne. Dès le lendemain, ce décalage temporel ne sera plus minimisé ${ }^{61}$, mais au contraire maximisé, toujours au profit du journal. L'héroïsation de celui-ci est validée par les autres, qui parlent d'i un " rapport " dévoilé ce vendredi $~^{62}$, d'une " révélation $\|^{63}$, d'une " exhumation " $^{64}$ ou de textes " déterrés des limbes du site Internet du Premier ministre, où ils dormaient depuis un mois déjà $"{ }^{65}$. Chacun fait comme si l'information avait été cachée, tout en reprochant au gouvernement ॥ l'erreur " d'avoir publié les rapports.

Dans les médias d'opinion, la logique du soupçon a un registre plus nettement politicien, le flou supposé volontaire devant servir de " ballon d'essai " ${ }^{66}$, de " galop d'essai offert aux partisans de la thèse de Monsieur Tuot et d'une partie de l'extrême gauche "I, écrit Corinne Lepage ${ }^{67}$, sans parler de l'argument de l'UMP, largement repris, d'un retour de la stratégie mitterrandienne de "I faire monter le Front national " pour affaiblir la droite. Ainsi, pris entre un schéma complotiste et l'hérö̈sation journalistique, certains médias iront jusqu'à

59. KOVACS, Stéphane, "Intégration: Jean-Marc Ayrault veut déconstruire les stéréotypes", www.lefigaro.fr, 11 février 2014.

60. Ibidem.

61. Sauf pour quelques médias qui continuent à parler d'un rapport « remis aujourd'hui au Premier ministre " (CLERIMA, Ludovic, "Intégration : la France va-t-elle enseigner le bambara à l'école ?", fr.myeurop.info, 13 décembre 2013), «publié vendredi matin » (JUHEL, Chloé, "L'intégration, nouvelle épine dans le pied de Hollande", www.lecourrierdelatlas.com, 16 décembre 2013) ou "remis sur son bureau le 13 décembre 2013» (JUHEL, Chloé, "Le gouvernement tourne le dos à l'intégration”, www.lecourrierdelatlas.com, 23 décembre 2013).

62. PHAM-LÊ, Jérémie, "Intégration : 'Ce rapport part du principe que la France est coupable et raciste'”, www.lexpress.fr, 13 décembre 2013 ; "Intégration : un rapport préconise le retour du voile à l'école", www.bienpublic.com, 13 décembre 2013; "Un rapport remet en cause la politique d'intégration", www.capital.fr, 13 décembre 2013.

63. "Vives réactions après l'exhumation d'un rapport sur l'intégration", www.liberation.fr 13 décembre 2013.

64. NIAKATE, Haby, "France: polémique autour du rapport d'intégration", www.jeuneafrique.com, 2 janvier 2014.

65. LEMONNIER, Marie ; MARTIN, Julien, "Intégration : comment Ayrault s'est pris les pieds dans le voile", tempsreel.nouvelobs.com, 22 décembre 2013 ; DEPRIECK, Matthieu, "Le rapport sur l'intégration fait dérailler la belle mécanique de Hollande", www.lexpress.fr, 17 décembre 2013.

66. CRAPEZ, Marc, "Les dessous du rapport sur l'intégration", www.contrepoints.org, 16 décembre 2013 ; LOZÈS, Patrick, "Flou sur le voile à l'école : le gouvernement en apprentisorcier", www.huffingtonpost.fr, 16 décembre 2013.

67. LEPAGE, Corinne, "Laïcité : avoir le courage de regarder en face la problématique de la relégation sociale”, www.huffingtonpost.fr, 16 décembre 2013. 
dire que l'information sur la réunion interministérielle officiellement annoncée pour janvier 2014 "avait fuité $\|^{68}$. On prépare donc le terrain à l'idée qu'un complot se tramait dans les couloirs des ministères jusqu'à ce que les médias le percent à jour.

La majoration du rôle médiatique va de pair avec une disqualification des rapports et des auteurs. S'il reprend au départ le terme administratif de "personnalités qualifiées " (une fois), Le Figaro impose surtout un discours sur les " experts" (cinq fois dans le dossier) et leur " jargon " : "Les experts [écrivent] de façon tortueuse " ${ }^{69}$. "Tortueuse "I, donc non pas compliquée, mais hypocrite, sournoise ou machiavélique, selon les synonymes.

Ce thème de l'intention cachée et/ou de la fourberie du procédé, qui confère inversement à la presse le rôle de "chevalier blanc" de l'information, est promis à un bel avenir. En effet, alors que certaines voix émergent pour pointer un II rapport déjà enterré et que personne n'a lu $11^{70}$, des commentateurs balaient l'idée même de lire la source, en en critiquant la longueur (" 250 pages ! ") et surtout le langage : " un jargon relevant du volapük " ${ }^{71}$, " une langue aussi bizarre qu'ampoulée qui mixte tous les défauts du charabia associatif, du jargon universitaire et du sabir technocratique "I, écrit le journaliste Jean-Marcel Bourguereau $^{72}$. Et les blogs de droite de renchérir : du "Volapük victimisant $\|^{73}$, un $"$ jargon prétentieux $\|^{74}$, " un jargon administratif inadapté et un lexique des sciences sociales venu essentiellement de l'anglais $"{ }^{75}$. "L Le rapport est écrit dans un langage abscons, caractère

68. JUHEL, Chloé, "Un rapport 'coup de pied' dans la fourmilière de l'intégration", www.lecourrierdelatlas.com, 13 décembre 2013; BAILLY, Guillaume, "L'intégration au cimetière, ou le cimetière de l'intégration", www.funeraire-info.fr, 18 février 2013 ; "Rapport sur l'intégration : l'appeau à trolls du PS fonctionne bien !", leparisienliberal.fr, 14 décembre 2013.

69. KOVACS, Stéphane, "Assumer la dimension 'arabe-orientale' de la France”, www.lefigaro.fr, 12 décembre 2013.

70. DELAPORTE, Lucie, “Intégration': ce rapport déjà enterré et que personne n'a lu”, www.mediapart.fr, 18 décembre 2013 ; Juan, "Intégration : Hollande face à la dernière manipulation du Figaro", www.paperblog.fr, 16 décembre 2013.

71. MONTVALLON, Dominique de ; HOUCHARD, Béatrice, "L'incroyable histoire du vrai-faux rapport sur l'intégration", www.lopinion.fr, 15 décembre 2013.

72. BOURGUEREAU, Jean-Marcel, "Intégration, le rapport qui divise", www.larepubliquedespyrenees.fr, 14 décembre 2013.

73. CRAPEZ, Marc, "Rapport sur l'intégration: parlez-vous le Volapük victimisant?", www.contrepoints.org, 24 décembre 2013.

74. CRAPEZ, Marc, "Les dessous du rapport sur l'intégration”, www.contrepoints.org, 16 décembre 2013.

75. BOUVET, Laurent, "Intégration : mettons les pieds dans le plat !", www.slate.fr/tribune, 21 décembre 2013. 
propre à la novlangue. C'est un mélange d'amphigouris sociologique et de franche idéologie $1{ }^{76}$.

Le dictionnaire nous dit que les termes "jargon", "amphigouri", "sabir" et "charabia" renvoient à un langage volontairement confus et incompréhensible, langue de malfaiteurs et/ou mâtinée de lexiques étrangers ou de patois, tandis que les termes "novlangue" et "volapük" évoquent un néo-langage, celui du monde totalitaire de $1984^{77}$ ou la langue universelle précédant l'espéranto.

Au-delà donc d'une critique visant la forme, que l'on trouve par ailleurs ${ }^{78}$, le choix de ces termes est significatif : ils pointent tous vers les figures de l'étranger, des intellectuels de gauche et des malfaiteurs supposés s'attaquer sournoisement aux valeurs nationales au bénéfice d'un totalitarisme, et détournant pour ce faire la propriété commune ici incarnée par la langue de la nation : "Le goulag postmoderne a son langage "I, écrit par exemple Frédéric Laboulaye ${ }^{79}$ sur le blog Nouvelles de France ${ }^{80}$; "l une action de rééducation des masses, par l'imposition d'une sorte de novlangue "I renchérit le site agoravox ${ }^{81}$.

Implicitement se déploie le fantasme de l'u islamo-gauchisme $\|^{82}$, qui veut que sous la promotion d'un "multiculturalisme", présumé typiquement étranger et favorable aux "islamistes", la gauche développerait son programme aux " aspects orwelliens ou soviétiques " ${ }^{83}$, menaçant une identité nationale elle-même synonyme de liberté. La scène est donc disposée comme un affrontement, un antagonisme binaire entre "modèle français" (nationaliste) et "modèle étranger" (dit II communau-

76. LABOULAYE, Frédéric, "Hollande et Ayrault ou la guerre civile tranquille", www.ndf.fr, 13 décembre 2013.

77. ORWELL, George, 1984, Paris : Éd. Gallimard, 2007, 407 p.

78. Une forme « ratée (276 pages compilées sans la moindre harmonisation)» (NIAKATE, Haby, "France : polémique autour du rapport d'intégration", www.jeuneafrique.com, 2 janvier 2014); "les textes ne sont pas assez édités pour être rendus digestes» (PROUTEAU, Thomas, "Le rapport sur l'Intégration, une parano et un énorme gâchis", www.slate.fr, 19 décembre 2013) ; des "formulations parfois un peu hasardeuses" (CROSNIER, Camille, "Intégration: un rapport envisage une refonte du système", www.rtl.fr, 13 décembre 2013 ).

79. Pseudonyme d'Olivier Pichon, enseignant, ancien élu Front national, proche des mouvements monarchistes.

80. LABOULAYE, Frédéric, "Hollande et Ayrault ou la guerre civile tranquille", www.ndf.fr, 13 décembre 2013.

81. Clémentine, "Rapport choc sur l'intégration: délire ou révolution?", www.agoravox.tv, 15 décembre 2013.

82. HAJJAT, Abdellali; MOHAMMED, Marwan, Islamophobie: comment les élites françaises fabriquent le problème musulman, Paris : Éd. La Découverte, 2013, 190 p.

83. CRAPEZ, Marc, "Les dessous du rapport sur l'intégration", www.contrepoints.org, 16 décembre 2013. 
tariste 11). Les rapports sont d'office renvoyés au communautarisme. Marc Leplongeon, journaliste du Point, veut voir dans la diffusion des "langues africaines " à l'école le "risque [...] d'exacerber des communautarismes $"{ }^{84}$. Pour le journal L'Opinion ou Le Parisien, les rapports " débouche[nt], mine de rien, sur une apologie du modèle communautariste anglo-saxon $1{ }^{85}$. Le chef de l'État et le Premier ministre sont obligés de s'en défendre : "Ma culture, ce n'est pas le multiculturalisme ou le communautarisme 11 , déclare Jean-Marc Ayrault ${ }^{86}$. Même sous la critique de forme pointe une peur obsessionnelle, cachée dans le consensus sur l'u intégration républicaine II. II y a là comme une boîte à ne pas (r)ouvrir, et c'est là l'enjeu de la dramatisation.

\section{L'autorisation du racisme}

Pour construire la menace, Le Figaro convoque deux discours politiques directement intéressés à cette logique, qui vont dire de manière flamboyante ce que la journaliste Stéphane Kovacs a surtout suggéré. C'est, d'une part, la tribune offerte à l'essayiste Malika Sorel-Sutter (campée en "spécialiste de l'intégration", mais qui vient de perdre sa position institutionnelle avec la fin du Haut Conseil à l'intégration, en 2013) et, d'autre part, la reprise du thème dans l'univers des blogs par le journaliste du Figaro Yves Thréard.

Dans la continuité du discours catastrophiste qui a servi l'entreprise de politisation de la laïcité ${ }^{87}$, Malika Sorel-Sutter traite le débat sous un angle nettement guerrier. Empruntant implicitement aux mythes de "l'islamisation de la France " ${ }^{88}$ et de sa " soviétisation " par la gauche, elle tient la coloration inclusive des rapports pour le signe d'une stratégie complotiste contre la nation et y oppose une lecture de l'intégration fondée en réalité sur " une racialisation de la "francité" ${ }^{89}$.

84. LEPLONGEON, Marc, "Rapport sur l'intégration: 'On sert la soupe aux islamistes'”, www.lepoint.fr, 17 décembre 2013.

85. MONTVALLON, Dominique de; HOUCHARD, Béatrice, "L'incroyable histoire du vrai-faux rapport sur l'intégration", www.lopinion.fr, 15 décembre 2013 ; VERNET, Henri, "Intégration : $67 \%$ des Français opposés à la discrimination positive”, www.leparisien.fr, 7 février 2014.

86. AMAR, Cécile, "Le rapport qui embarrasse l'exécutif", www.lejdd.fr, 15 décembre 2013.

87. LORCERIE, Françoise, "La 'loi sur le voile' : une entreprise politique”, Droit et Société, $n^{\circ} 68$, 2008, pp. 53-74.

88. LIOGIER, Raphaël, Le mythe de l'islamisation: essai sur une obsession collective, Paris : Éd. du Seuil, 2012, 212 p.

89. SAFI, Mirna, "Une refondation manquée : les politiques d'immigration et d'intégration en France", www.laviedesidees.fr, 2014. 
Le propos mérite d'être largement cité tant il annonce et condense les grands thèmes qui structurent toute l'entreprise politique de construction de la menace : "L La rupture avec l'héritage du peuple français est pleinement assumée. Rien n'est laissé au hasard, comme en témoignent les recommandations qui détaillent les modalités de la rééducation des masses. II faut traquer les Français de souche culturelle européenne et leur propension à discriminer l'autre, en mettant sur pied de nouvelles institutions, ainsi qu'une multitude de mesures dignes d'une véritable police de la pensée. Même la langue française voit sa suprématie contestée! $\grave{A}$ aucun moment on ne trouve l'expression d'une quelconque reconnaissance de dette pour tout ce que la France a pu donner aux étrangers extraeuropéens et à leurs enfants. Le nombre de fois où apparaît le mot "devoir" est révélateur de l'esprit du texte : 13 fois seulement, tandis que le mot "droit" est cité 440 fois ! [...]. II faut se souvenir ici du rapport de Terra Nova [...]. L'électorat y était passé au scanner [...]. II est manifeste que cette refondation de la politique d'intégration n'est pas innocente. Elle nourrira le ressentiment des Français, avec une traduction probable dans les urnes... [...]. À quelques exceptions près, la plupart des membres sont inconnus sur les sujets traités. Mais il ne faut pas accorder trop d'importance à la composition des groupes, qui m'apparaissent comme de simples cautions : l'entreprise était déjà décidée $11^{90}$.

Référence à un suprémacisme " de souche culturelle européenne " qui serait menacé ; " hystérisation des marques d'appartenance politicoreligieuses $1{ }^{91}$, ici la langue et le voile ; référence au rôle "civilisateur" de l'intégration, avec le thème de la " dette " des " étrangers extraeuropéens " (sorte d'écho au thème du "rôle positif de la colonisation" des années 2000); retournement de la critique en normalisation (qui, dans les rapports, visait l'"intégration") dans la référence maoïste de la " rééducation des masses ", après Michèle Tribalat, qui parlait déjà de " reformatage ", de " redressement idéologique " et de "rénovation des mentalités $1{ }^{92}$; logique disciplinaire ${ }^{93}$ opposant les " devoirs " au droit ; abaissement de la qualité des membres des groupes de travail et du sens du travail ; dénonciation d'une stratégie électoraliste "communautariste" supposée orchestrée par la boîte à idées Terra Nova...

90. KOVACS, Stéphane, "Malika Sorel-Sutter : “Une véritable police de la pensée’”, www.lefigaro.fr, 12 décembre 2013.

91. BALIBAR, Étienne, "Dissonances dans la laïcité", Mouvements, $\mathrm{n}^{\circ} 33-34$, mai-juillet 2004 , pp. 148-161.

92. TRIBALAT, Michèle, "Intégration : les 5 rapports qui poussent la France sur la voie du multiculturalisme choisi sans le dire trop haut", www.atlantico.fr, 9 décembre 2013.

93. FOUCAULT, Michel, Surveiller et punir : naissance de la prison, Paris : Éd. Gallimard, 1993, 340 p. 
Convergeant vers une dénonciation de "la préférence étrangère ", comme dit encore Malika Sorel ${ }^{94}$, tous ces thèmes sont rassemblés et reliés, tel un fourbi mis à disposition pour la bataille, et accompagnés d'une prophétie (auto-réalisatrice) : cette construction de la menace, en effet, "I nourrira le ressentiment des Français, avec une traduction probable dans les urnes $1{ }^{95}$, ce que montrent abondamment les commentaires des lecteurs. Cet ordre de discours est repris simultanément dans le blog d'Yves Thréard : "Négation nationale ", titre celui-ci, où la nation se confond dans la fierté supposée persécutée de "l'homme blanc et hétérosexuel " : "Comme les bons élèves, qui sont souvent la cible des railleries de leurs petits camarades, l'homme blanc et hétérosexuel pourrait bientôt être obligé, dans notre pays, de se cacher $1{ }^{96}$. En toile de fond, un terrain scolaire implicitement ethnicisé : ces "bons élèves" menacés, thème classique du discours sur les "banlieues"... Ce syncrétisme des arguments est d'autant plus efficace qu'il est bien installé dans le paysage médiatico-institutionnel, et régulièrement réamorcé de polémique en polémique (pain au chocolat vs ramadan, famille vs mariage pour tous, identité naturelle vs théorie du genre, etc.).

Le Figaro impose aussi la ligne principale de lecture : le II rapport qui veut autoriser le voile à l'école ") (la formule se retrouve dans $20 \%$ des articles d'information), " propose son retour $\|^{97}$, " recommande $\|^{98}$, " préconise $\|^{99}$, voire " encourage la réintroduction du hijab à l'école $\|^{100}$. Si de rares journaux parlent d'un "rapport sur l'immigration $\|^{101}$, la

94. DEVECCHIO, Alexandre, "Malika Sorel : 'Le pouvoir bascule dans la préférence étrangère'”, www.lefigaro.fr/vox, 6 février 2014.

95. KOVACS, Stéphane, "Malika Sorel-Sutter: 'Une véritable police de la pensée'”, www.lefigaro.fr, 12 décembre 2013.

96. THRÉARD, Yves, "La négation nationale", blog.lefigaro.fr/threard, 12 décembre 2013.

97. CROSNIER, Camille, "Intégration : un rapport envisage une refonte du système", www.rtl.fr, 13 décembre 2013 ; CLERIMA, Ludovic, "Intégration : la France va-t-elle enseigner le bambara à l'école ?", fr.myeurop.info, 13 décembre 2013 ; HOFFNER, Anne-Bénédicte, "Cinq rapports préconisant de 'repenser l'intégration' suscitent un tollé", www.la-croix.com, 13 décembre 2013 ; "Le rapport sur l'intégration : la polémique continue", www.jolpress.com, 29 décembre 2013 ; VERNET, Henri, "Intégration : $67 \%$ des Français opposés à la discrimination positive", www.leparisien.fr, 7 février 2014.

98. "Rapport sur l'intégration : Ayrault réfute tout couac", tempsreel.nouvelobs.com, 16 décembre 2013.

99. REYNE, Florent, “Un rapport pour refonder l'intégration”, www.respectmag.com, 23 décembre 2013 ; "Un rapport choc préconise d'autoriser le voile à l'école", journaldumusulman.fr, 13 décembre 2013.

100. MICHKET, Oum, "Un rapport demandé par le Gouvernement préconise le retour du hijab à l'école", www.ajib.fr, 13 décembre 2013.

101. HAMCHICHE, Imene, "Intégration : le rapport qui divise", movil.ibercampus.fr, 14 décembre 2013 ; "Rapport gouvernemental sur l'immigration: ils sont devenus complètement fous, l'intégration des immigrés a échoué, alors ils veulent intégrer les Français", prorussia.tv, 18 décembre 2013. 
question majeure est celle du rapport entre islam et nation, symbolisée par l'antagonisme supposé entre "le voile" et "l'école". " La colère vient principalement de la remise en question de l'école républicaine. Parmi les idées évoquées, le retour du port du voile dans les écoles semble cristalliser toutes les frayeurs ${ }^{102}$. Dans une enquête à charge, le site Arrêt sur images interroge : "Dans la galaxie des chercheurs, Dhume est-il connu pour des prises de position provoile ? [...]. Khalid Hamdani, de son côté, était déjà connu pour avoir défendu le voile au moment de la commission Stasi $1{ }^{103}$.

L'obsession sous-jacente de l'"identité nationale", à laquelle l'on oppose "le voile", est l'aune à laquelle on mesure la respectabilité des auteurs. Les médias d'opinion racisent ouvertement la lecture : des sites de la galaxie nationaliste et islamophobe - qui relie droite catholique, extrême droite, pro-lsraël, gauche laïcarde, voire révolutionnaire ${ }^{104}$, par exemple Nouvelles de France, Le Nonce, Riposte laïque, Boulevard Voltaire, La-meilleure.com, messianique.forumpro, Israëlflash, etc. - présentent " Khalid Magdani " [sic] comme " sociologue marocain "l et lui inventent une (dis)position anti-chrétienne : "Un des auteurs du "rapport sur l'intégration" veut interdire de faire sonner les cloches $11^{105}$. On invente des prétextes pour se convaincre de soupçonner l'œuvre d'une main étrangère : "Mais quel pays les paie pour détruire la France ! $"{ }^{106}$. II y a donc clairement une continuité entre les entrepreneurs politiques initiaux et le traitement médiatique dans son ensemble, puis avec la réaction des lecteurs; les variations observables relèvent moins d'un désaccord que d'une forme de partage des tâches et d'une gradation des arguments disqualifiants : chaque média, selon sa sensibilité et sa position dans le champ, valorise plutôt certains arguments que d'autres parmi le stock initial. Mais le schéma est identique.

102. CLERIMA, Ludovic, "Intégration: la France va-t-elle enseigner le bambara à l'école ?", fr.myeurop.info, 13 décembre 2013.

103. DAUSSY, Laure, "Matignon et le rapport sur l'intégration: autopsie d'un cafouillage", www.arretsurimages.net, 17 décembre 2013.

104.PARTI COMMUNISTE MARXISTE LÉNINISTE MAOÏSTE, “Le rapport 'post-moderne' sur l'intégration", lesmaterialistes.com, 15 décembre 2013.

105. JANVA, Michel, "Un des auteurs du 'rapport sur l'intégration' veut interdire de faire sonner les cloches", lesalonbeige.blogs.com, 19 décembre 2013 ; "Rapport sur l'intégration : comment 'continuer à accepter sans réagir que les cloches sonnent tous les dimanches' ?", www.ndf.fr, 19 décembre 2013 ; OBSERVATOIRE DE L'ISLAMISATION, "Pour un auteur du 'rapport sur l'intégration' (salué par Ayrault) les 'républicains laïques et cohérents' devraient réduire au silence les cloches des églises", www.islamisation.fr, 19 décembre 2013.

106. BEYLAU, Pierre, "Intégration : alerte aux pyromanes, lepoint.fr, 13 décembre 2013. 


\section{L'intimidation}

Dans la foulée, les politiques en appellent à II un sursaut républicain collectif $1{ }^{107}$. Les médias d'opinion rendent explicites les sous-entendus ethnonationalistes, dénigrant les auteurs pour s'inscrire dans le "consensus républicain": " De véritables apprentis sorciers qui veulent mettre à mal l'un des fondements même de notre République I", écrit le député UMP Sébastien Huyghe ${ }^{108}$, " entourés de simples étudiants ou de diverses associations de défense des immigrés $1{ }^{109}$. Avec des variations d'intensité selon les positions dans le champ, un discours d'insulte se donne libre cours, porté par le consensus global et explicité par les médias d'opinion. Sont dénoncés des "révisionnistes dynamiteurs", " docteurs Folamour [qui] ont rédigé leur pensum " ${ }^{110}$ ou des " cerveaux malades de Bobos pervers $)^{111}$; on propose d'en faire la liste infâme sur un "Mur des Cons. II faudra remplacer "cons" par un qualificatif plus précis et moins sympathique ${ }^{112}$.

La disqualification ultime se joue sur le plan raciste et islamophobe. Le soupçon sur la qualité de "personnalités qualifiées? " ${ }^{113}$ — ces qualifiés $\|^{114}$ — et sur le "fameux rapport de 276 pages des 250 plus grands experts sur l'intégration $1{ }^{115}$ a un implicite raciste et islamophobe que les sites de la galaxie nationaliste ne font qu'expliciter. II Je propose qu'Olivier Noël renonce à son patronyme fleurant trop bon la chrétienté et se mette à la page, Olivier Ramadan serait bien plus de mise dorénavant $" 1{ }^{116}$. En présentant la "liste des "experts"

107. GUIROUS, Lydia, "Laïcité inclusive: la France des Lumières brûle", www.huffingtonpost.fr, 13 décembre 2013.

108. HUYGHE, Sébastien, "Rapport sur l'intégration: une feuille de route inacceptable", sebastienhuyghe.blogs.com, 13 décembre 2013,

109. Forum, "Les patriotes avec Marine, 2014-2017", www.marinelepenelysee2012.com ; "Un rapport gouvernemental préconise d'assumer la dimension 'arabe-orientale' de la France", www.defrancisation.com, 13 décembre 2013.

110. BEYLAU, Pierre, "Intégration : alerte aux pyromanes", www.lepoint.fr, 13 décembre 2013.

111. "Rapport gouvernemental sur l'immigration : ils sont devenus complètement fous, l'intégration des immigrés a échoué, alors ils veulent intégrer les Français", www.prorussia.tv, 18 décembre 2013.

112. "Rapport sur l'intégration : des noms !", grincheux.typepad.com, 15 décembre 2013.

113. BEYLAU, Pierre, "Intégration : alerte aux pyromanes”, www.lepoint.fr, 13 décembre 2013.

114.CONSIGNY, Charles, "Consigny : ce que dit vraiment le rapport sur l'intégration", www.lepoint.fr, 16 décembre 2013.

115. LATINA, Jean-Pierre, "Le fameux rapport de 276 pages des 250 plus grands experts sur l'intégration pour la favoriser en commençant par ne plus l'appeler intégration", fablehaikus.overblog.com, 16 décembre 2013.

116. “Rapport sur l'intégration : des noms !”, grincheux.typepad.com, 15 décembre 2013. 
ayant contribué au rapport sur l'intégration $1{ }^{117}$ sont effacés les noms vus comme "européens" - fussent-ils ceux d'auteurs des rapports et conservés seulement ceux vus comme "maghrébins". Et de commenter : "Que des "chances pour la France" — AUCUN RACISME ni soupçon d'incompétence, juste le constat $"{ }^{118}$. "Demandez-vous pourquoi ce fameux rapport sur l'intégration est tellement favorable aux immigrés de tous poils? Et allez voir plus bas sur la liste des "EXPERTS"... II n'y a pas un nom de Français! On demande à des imams, à des assos pourvoyeuses d'aides sociales pro-immigrationnistes... $1{ }^{119}$.

Certains sites complètent l'hystérisation raciste avec une menace voilée : "Les inspirateurs d'un tel rapport sont des fauteurs de guerre civile. Ils devront être jugés, à tout le moins électoralement, pour ce qu'il faut bien appeler une trahison $11^{120}$. En fournissant les adresses des rédacteurs principaux des rapports on incite discrètement à l'action. Comme suite logique, des auteurs, mais également certains des conseillers des cabinets ministériels impliqués ${ }^{121}$, sont destinataires de mails et lettres anonymes. Les courriers que nous avons nous-même reçus à titre personnel - à notre domicile, à notre travail sous le sceau " Personnel. Faire suivre " et jusque dans des lieux d'intervention publique - sont éclairants sur le rôle des médias en ce qu'ils reposent sur l'envoi compilé, souligné et commenté d'extraits de blogs (Nouvelles de France, Riposte laïque, Boulevard Voltaire...), d'articles ou d'ouvrages légitimant des discours islamophobes (comme C'était de Gaulle ${ }^{122}$ ), ou censés démontrer le danger de l'"islam" (extraits du Coran, d'un " article écrit par un journaliste d'origine musulmane ", etc.). Ils oscillent entre un discours sur la menace et un discours de la menace, comme ce dessin d'un gibet et d'une croix de Lorraine ainsi commenté : "Où

\footnotetext{
117. FRANCAISDEFRANCE, "La liste des 'experts' ayant contribué au rapport sur l'intégration du gouvernement Ayrault", www.la-meilleure.com, 24 décembre 2013 ; DICKÈS, Jean-Pierre, "Retour sur le 'Rapport sur l'intégration'”, media-presse.info, 5 janvier 2014.

118. CHAHBI-BOUILLON, Inès, "Intégration: le rapport choc... 440 droits, 13 devoirs...", unionrepublicaine.fr, 22 décembre 2013.

119. FRANCAISDEFRANCE, "La liste des 'experts' ayant contribué au rapport sur l'intégration du gouvernement Ayrault", www.la-meilleure.com, 24 décembre 2013. Cette liste biaisée circule de site en site ; on la retrouve postée par des lecteurs jusque sur celui de BFMTV : www.bfmtv.com/ economie/municipales-couteront-118-millions-aux-contribuables-683560/avis, consulté le 2 juillet 2014.

120. LABOULAYE, Frédéric, "Hollande et Ayrault ou la guerre civile tranquille", www.ndf.fr, 13 décembre 2013.

121. Nous n'avons pas d'information systématique permettant d'aller plus avant dans l'analyse de cette dimension du processus ; cela concerne au moins la moitié des rédacteurs et plusieurs membres de cabinets ministériels.

122. PEYREFITTE, Alain, C'était de Gaulle, Paris : Éd. Fayard, 1995, 893 p.
} 
qu'ils SOIENT, quoi qu'ils FASSENT, les TRAÎTRES seront CHÂTIÉS ${ }^{123}$. La logique d'intimidation s'autorise d'un imaginaire de la Résistance nationale passé peut-être par d'autres étapes.

\section{Statu quo}

La polémique a-t-elle échappé à ses entrepreneurs politiques? Nous avons montré que les ressorts thématiques et argumentaires de la disqualification furent d'emblée réunis et mis à disposition par les initiateurs, soit une alliance entre Le Figaro, des intellectuels anciens membres du Haut Conseil à l'intégration (Michèle Tribalat, Malika Sorel, puis Guylain Chevrier, Jean-Philippe Moinet...) et peut-être l'UMP : Le Nouvel Observateur note que II la concomitance est troublante : cette même semaine, le jeudi, l'UMP tenait une convention sur... l'immigration ! [...]. Ce n'est pas la première fois que le quotidien de Dassault se fait le porte-voix de l'UMP. L'opération n'en reste pas moins efficace $1{ }^{124}$. La dynamique qui s'en est suivie fut celle de la recirculation ${ }^{125}$ des thèmes de disqualification, avec un enflement dans toutes les directions et sous diverses formes.

Cela est certes lié à la concurrence entre médias et au fait que la passion des thèmes centraux de la polémique ("intégration", "école", "islam"...) oblige à prendre position d'une manière qui se démarque. Mais l'exceptionnelle continuité du discours entre les émetteurs, l'hystérisation du thème et le grotesque des formes indiquent en même temps un emballement dans la mobilisation pour le maintien hégémonique du discours moral de l'"intégration" (l'insistance sur les devoirs plutôt que sur le droit en est un symbole).

L'enjeu de la constitution des rapports en menace pour la "nation" et la "République" était de faire "enterrer" des rapports publics qui accréditent des voix minoritaires - comme l'a relevé Esther Benbassa ${ }^{126}$ comme si un autre discours que celui de l'"intégration" allait faire littéralement disparaître la société française. Le registre discursif du funéraire permet une mise en équivalence dramatique, comme si de l'"enterrement" des rapports dépendait la survie de la "nation" con-

123. Extrait d'un des courriers anonymes reçus.

124. LEMONNIER, Marie ; MARTIN, Julien, “Intégration : comment Ayrault s'est pris les pieds dans le voile", tempsreel.nouvelobs.com, 22 décembre 2013.

125. BUTLER, Judith, Le pouvoir des mots : discours de haine et politique du performatif, Paris : Éd. Amsterdam, 2004, 219 p.

126. BENBASSA, Esther, "Quand les 'Arabes' prennent le pouvoir par la plume : ces rapports qui font trembler le gouvernement”, www.huffingtonpost.fr, 16 décembre 2013. 
fondue dans un "modèle d'intégration". C'est bien le sens de la pétition de l'Union nationale inter-universitaire (UNI), qui dénonce "I une trahison de notre identité, des principes et valeurs républicaines, et des règles qui seules permettent la cohésion nationale II et organise un cortège funéraire " contre le rapport d'intégration, fossoyeur de l'idée d'intégration " ${ }^{127}$.

Si l'excès fut logiquement le plus spectaculaire dans la galaxie nationaliste et islamophobe, la quasi-totalité des médias d'information, soutiens actifs du "consensus républicain", y ont contribué. Ils ont ensemble diffusé et entretenu l'alarme, tout en la contrôlant en partie. En effet, par la construction et la diffusion d"'informations" qui évitent de discuter de la source - le contenu réel des rapports - ils ont d'emblée écarté l'objet représentant la mésentente ${ }^{128}$ derrière le consensus. Gardiens scrupuleux d'une boîte noire, au motif qu'il s'agirait d'une " boîte de Pandore ", ils ont arbitré les voix et les places, renvoyant au besoin les protagonistes à un statut racial ou religieux afin " qu'on ne franchisse pas la ligne rouge 11, comme l'écrit $L^{\prime}$ Express ${ }^{129}$.

Les médias ont veillé à ce que chacun reste à sa place, selon une conception dominante de l'intégration exprimée par un lecteur du Monde : "À Rome, fais comme les Romains... " ${ }^{130}$. Pour appuyer ce réflexe de propriétaire et justifier qu'on ne discute pas de l'ordre et des problèmes de la cité, les médias ont mobilisé des sondages, comme Le Parisien trouvant $77 \%$ de répondants pour affirmer que " ce sont avant tout les personnes étrangères qui doivent se donner les moyens de s'intégrer $1{ }^{131}$. Ils ont également convoqué les représentants politiques sur ce terrain, recherchant principalement auprès de ceux de la gauche et du gouvernement leur opposition aux rapports comme à la stratégie du Premier ministre : " Hollande enterre " ${ }^{132}$, " Fabius désapprouve $\|^{133}$, "Manuel Valls [...] a condamné $\|^{134}$, "le premier

127. Union nationale inter-universitaire, http://www.uni.asso.fr/spip.php?article10992, consulté le 2 juillet 2014.

128. RANCIÈRE, Jacques, La mésentente : politique et philosophie, Paris : Éd. Galilée, 1995, 187 p.

129. PHAM-LÊ, Jérémie, "Intégration : 'Ce rapport part du principe que la France est coupable et raciste'”, www.lexpress.fr, 13 décembre 2013.

130. LE BARS, Stéphanie, "Revenir sur le voile à l'école : une proposition iconoclaste et inutile ?", www.lemonde.fr, 13 décembre 2013.

131. VERNET, Henri, "Intégration: $67 \%$ des Français opposés à la discrimination positive", www.leparisien.fr, 7 février 2014.

132. "Un rapport choc préconise d'autoriser le voile à l'école", journaldumusulman.fr, 13 décembre 2013 ; "Hollande enterre le rapport sur l'intégration", www.ledauphine.com, 13 décembre 2013.

133. “Laurent Fabius désapprouve le rapport sur l'intégration”, www.capital.fr, 15 décembre 2013.

134. MONTVALLON, Dominique de; HOUCHARD, Béatrice, "L'incroyable histoire du vrai-faux rapport sur l'intégration”, www.lopinion.fr, 15 décembre 2013. 
secrétaire du Parti socialiste prend ses distances $\|^{135}$, "Royal tacle la "méthode" Matignon $1{ }^{136}$.

Dans ce jeu médiatique, se démarquer politiquement veut dire se démarquer des rapports. Manuel Valls fut l'un des premiers, empruntant la rhétorique de la droite : "Dans les propositions de ces groupes de travail, il y a des aberrations et des propositions dangereuses qui mettent en cause ce qu'est la France, sa laïcité $1{ }^{137}$.

Ce qui s'est joué n'a donc que peu à voir avec les rapports en eux-mêmes. L'enjeu est plutôt le maintien sans discussion d'une représentation de la nation comme un "chez-nous-bien-de-chez-nous". La polémique a ainsi fonctionné sur une projection - au sens fantasmatique du terme - et selon un enjeu d'apparence : la réaction à ce qu'on en dit. Prenons l'exemple du ministre Laurent Fabius déclarant : "l Le rapport, je ne l'ai pas lu, i'en ai pris connaissance par les journaux, et en le lisant, je pensais à une phrase que j'aimais beaucoup de François Mitterrand, qui disait : "Il ne faut pas prendre toutes les mouches qui volent pour des idées" $1{ }^{138}$. L'enjeu pour lui était alors de dédramatiser, et même de prendre à la légère, nullement d'entrer dans le débat; et pour cela, la lecture du Figaro a suffi.

De fait, le débat n'a pas eu lieu. L'entreprise politique a finalement eu raison de cette initiative progressiste, en entraînant dans le sillage du Figaro et de ses alliés les positions de la plupart de ceux qui ont pris publiquement la parole : à la stratégie initiale relayée par toute la presse ou presque a répondu l'aboiement des politiciens de droite, puis la division de ceux de gauche entre alignement sur la polémique et renoncement au projet.

L'initiative du Premier ministre de l'époque a été littéralement mise hors-circuit, comme une anticipation médiatiquement orchestrée de sa mise à l'écart politique au profit de Manuel Valls. Cet épisode a montré que, dans le registre de la représentation médiatique et politique de la société, tout est bon pour dramatiser ce qui en symbolise la pluralisation morale concrète et ainsi conserver l'hégémonie du

135. “Désir : l'interdiction du voile à l'école pas remise en cause”, www.bfmtv.com, 15 décembre 2013.

136. ROVAN, Anne, "Rapport sur l'intégration : Royal tacle la 'méthode' Matignon", www.lefigaro.fr, 16 décembre 2013; "Rapport sur l'intégration: Ayrault réfute tout couac", tempsreel.nouvelobs.com, 16 décembre 2013.

137. AMAR, Cécile, "Le rapport qui embarrasse l'exécutif”, www.lejdd.fr, 15 décembre 2013.

138. “Laurent Fabius désapprouve le rapport sur l'intégration”, www.capital.fr, 15 décembre 2013 ; "Le rapport sur l'intégration est-il déjà enterré ?", www.francetvinfo.fr, 15 décembre 2013. Le gras est de notre fait. 
discours ethnonationaliste sur I'“intégration". L'anathème lancé sur les rapports fait certes écho à la sourde angoisse civilisationnelle de l'époque, d'où elle tire son registre proche de la "panique morale". Mais l'unitarisme idéologique qui se nourrit de cette angoisse se fait au prix du déni de la réalité de la France et de ses défis actuels, comme le rappelle clicanoo.re, le journal de la Réunion, en commentant "I des propositions qui n'ont rien d'original pour les Réunionnais, mais feraient forcément bouger les repères métropolitains si elles étaient appliquées $11^{139}$. 